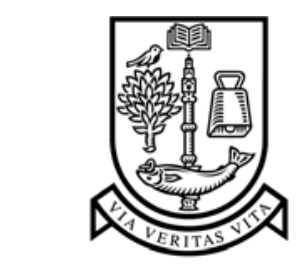

UNIVERSITY

of

GLASGOW

Mohammed, A. and Ould-Khaoua, M. and Mackenzie, L. (2007) An efficient counter-based broadcast scheme for mobile ad hoc networks. Lecture Notes in Computer Science 4748:pp. 275-283.

http://eprints.gla.ac.uk/3699/ 


\title{
An Efficient Counter-Based Broadcast Scheme for Mobile Ad Hoc Networks
}

\author{
Aminu Mohammed, Mohamed Ould-Khaoua, and Lewis Mackenzie \\ Department of Computing Science, University of Glasgow, G12 8RZ, \\ Glasgow, United Kingdom \\ \{maminuus, mohamed, lewis\} adcs.gla.ac.uk
}

\begin{abstract}
In mobile ad hoc networks (MANETs), broadcasting plays a fundamental role, diffusing a message from a given source node to all the other nodes in the network. Flooding is the simplest and commonly used mechanism for broadcasting in MANETs, where each node retransmits every uniquely received message exactly once. Despite its simplicity, it however generates redundant rebroadcast messages which results in high contention and collision in the network, a phenomenon referred to as broadcast storm problem. Pure probabilistic approaches have been proposed to mitigate this problem inherent with flooding, where mobile nodes rebroadcast a message with a probability $p$ which can be fixed or computed based on the local density. However, these approaches reduce the number of rebroadcasts at the expense of reachability. On the other hand, counter-based approaches inhibit a node from broadcasting a packet based on the number of copies of the broadcast packet received by the node within a random access delay time. These schemes achieve better throughput and reachability, but suffer from relatively longer delay. In this paper, we propose an efficient broadcasting scheme that combines the advantages of pure probabilistic and counter-based schemes to yield a significant performance improvement. Simulation results reveal that the new scheme achieves superior performance in terms of saved-rebroadcast, reachability and latency.
\end{abstract}

Keywords: MANETs, Flooding, Broadcast storm problem, Saved-rebroadcast, Reachability, Latency.

\section{Introduction}

Broadcasting is a means of diffusing a message from a given source node to all other nodes in the network. It is a fundamental operation in MANETs and a building block for most other network layer protocols. Several unicast routing protocols such as Dynamic Source Routing (DSR), Ad Hoc on Demand Distance Vector (AODV), Zone Routing Protocol (ZRP), and Location Aided Routing (LAR), as well multicast protocols employ broadcasting to detect and maintain routes in a dynamic environment. Currently, these protocols typically rely on simplistic form of broadcasting called simple flooding, in which each mobile node retransmits every unique received packet exactly once. Although flooding is simple and easy to 
implement, it often causes unproductive and harmful bandwidth congestion, a phenomenon referred to as the broadcast storm problem [1], [2], [3].

Several broadcast schemes have been proposed that mitigate the broadcast storms problem. The performance of these schemes is measured in terms of reachability, which is the fraction of the total nodes that receive the broadcast messages, the savedrebroadcast, that is the fraction of the total nodes that does not rebroadcast the messages, and the latency, that is the time between the first and the last instant that the broadcast message is transmitted [4]. These schemes are usually divided into two categories [4], [5]: deterministic schemes and probabilistic schemes. Deterministic schemes require global topological information of the network and are guaranteed a reachability of 1 considering an ideal MAC layer. However, they incur large overhead in terms of time and message complexity for maintaining the global knowledge requirements due to the inherent dynamic topology of MANETs. On the other hand, probabilistic schemes do not require global topological information of the network to make a rebroadcast decision. As such every node is allowed to rebroadcast a message based on a predetermined forwarding probability $p$. As a consequence, these schemes incur a smaller overhead and demonstrate superior adaptability in dynamic environment when compared to deterministic schemes [6]. However, they typically sacrifice reachability as a trade-off against overhead.

Among the probabilistic schemes that have been proposed are probability-based and counter-based schemes [1], [2], [3]. In probability-based schemes, a mobile node rebroadcasts a message according to certain probability $p$ which can be fixed or computed based on the local density. Current probabilistic schemes assume a fixed probability value and it is shown [1], [4], [7] that the optimal rebroadcast probability is around 0.65 . However, these approaches reduce the number of rebroadcast at the expense of reachability [2]. In contrast, messages are rebroadcast only when the number of copies of the message received at a node is less than a threshold value in counter-based schemes. This lead to better throughput and reachability, but suffer from relatively longer delay [3], [4].

In this paper, we proposed an efficient counter-based scheme that combines the advantages of probabilistic and counter-based schemes. We set a rebroadcast probability at each node (as in [1], [4] and [7]) if the packet counter is less than the threshold value rather than rebroadcasting the message automatically. This is because the packet counter is not exactly equal to the node number of neighbors. Otherwise we drop the message. We compare this scheme with simple flooding, fixed probability and counter-based scheme. Simulation results reveal that this simple adaptation can lead to a significant performance improvement.

The rest of the paper is organized as follows: In Section 2, we introduce the related work on probabilistic and counter-based schemes. The description of our scheme is presented in Section 3. We evaluate the performance of our scheme and present the simulation results in Section 4. Finally, concluding remarks are presented in Section 5.

\section{Related Work}

This section sheds some light on the research work related to probabilistic and counter-based broadcasting schemes. 
Ni et al [2] proposed a probability-based scheme to reduce redundant rebroadcast by differentiating the timing of rebroadcast to avoid collision. The scheme is similar to flooding, except that nodes only rebroadcast with a predetermined probability $P$. Each mobile node is assigned the same forwarding probability regardless of its local topological information. In the same work, counter-based scheme is proposed after analysing the additional coverage of each rebroadcast when receiving $n$ copies of the same packet.

Cartigny and Simplot [8] have proposed an adaptive probabilistic scheme. The probability $p$ for a node to rebroadcast a packet is determined by the local node density and a fixed value $k$ for the efficiency parameter to achieve the reachability of the broadcast. However, the critical question thus becomes how to optimally select $k$, since $k$ is independent of the network topology.

In $\mathrm{Ni}$ et al follow-on work [3], the authors have proposed an adaptive counterbased scheme in which each node dynamically adjusts its threshold value $C$ based on its number of neighbors. Specifically, they extend the fixed threshold $C$ to a function $C(n)$, where $n$ is the number of neighbors of the node. In this approach there should be a neighbor discovery mechanism to estimate the current value of $n$. This can be achieved through periodic exchange of 'HELLO' packets among mobile nodes.

Recently, Zhang and Agrawal [9] have described a dynamic probabilistic broadcast scheme which is a combination of the probabilistic and counter-based approaches. The scheme is implemented for route discovery process using AODV as base routing protocol. The rebroadcast probability $P$ is dynamically adjusted according to the value of the local packet counter at each mobile node. Therefore, the value of $P$ changes when the node moves to a different neighborhood; for example, in sparser areas, the rebroadcast probability is large compared to denser areas. To suppress the effect of using packet counter as density estimates, two constant values $d$ and $d_{l}$ are used to increment or decrement the rebroadcast probability. However, the critical question is how to determine the optimal value of the constants $d$ and $d_{l}$.

In this paper, we propose an efficient counter-based scheme which combines the merits of probability-based and counter-based algorithms to yield a significant performance improvement in terms of saved rebroadcast, reachability and end-to-end delay which are simple enough for easy implementation. The detail of the scheme is described in the next section.

\section{Efficient Counter-Based Scheme (ECS)}

In this section, we present the efficient counter-based scheme that aims to mitigate the broadcast storm problem associated with flooding. The use of ECS for broadcasting enables mobile nodes to make localized rebroadcast decisions on whether or not to rebroadcast a message based on both counter threshold and forwarding probability values. Essentially, this adaptation provides a more efficient broadcast solution in sparse and dense networks.

In ECS, a node upon reception of a previously unseen packet initiates a counter $c$ that will record the number of times a node receives the same packet. Such a counter is maintained by each node for each broadcast packet. After waiting for a random assessment delay (RAD, which is randomly chosen between 0 and $\mathrm{T}_{\max }$ seconds), if $c$ 
reaches a predefined threshold $C$, we inhibit the node from this packet rebroadcast. Otherwise, if $c$ is less than the predefined threshold, $C$, the packet is rebroadcast with a probability $P$ as against automatically rebroadcasting the message in counter-based scheme. The use of a rebroadcast probability stem from the fact that packet counter value does not necessarily correspond to the exact number of neighbours of a node, since some of its neighbours may have suppressed their rebroadcast according to their local rebroadcast probability. Thus, the selection of an optimal forwarding probability is vital to the performance of our scheme. Based on [1], [4], and [7], we opt for a rebroadcast probability of 0.65 . A snapshot of our algorithm is presented in figure 1 .

\section{Performance Analysis}

This section studies the performance of our scheme, counter-based, fixed probability and flooding in terms of reachability, saved-rebroadcast and latency. In order to isolate the effects of various design choices of the broadcast algorithms on performance we do not simulate other protocol layers such as the MAC and physical layers. Our performance analysis is based on the assumptions widely used in literature [11], [12], [17].

i. All nodes participate fully in the protocol of the network. In particular each participating node should be willing to forward packets to other nodes in the network.

\section{Algorithm: Efficient Counter-Based Scheme}

On hearing a broadcast message $m$ at a node $X$

- $\quad$ initialize the counter $\mathrm{c}=1$;

- $\quad$ set and wait for RAD to expire;

- for every duplicate message $m$ received within RAD

$\circ$ increment $\mathrm{c}, \mathrm{c}=\mathrm{c}+1$;

0 if $(\mathrm{c}<\mathrm{C})$ (counter threshold-value) \{

- wait for RAD to expires;

- rebroadcast probability $P=P_{l}$; $\}$

else $\left\{\right.$ //where $P_{1}=0.65$

- stop waiting

- Drop the message $\}$

- Generate a random number $R N$ over $[0,1]$

- If $R N \leq P$

$$
\text { else }
$$

○ Drop the message

Fig. 1. A snapshot of efficient counter-based scheme algorithm 
ii. Packet may be corrupted or lost in the wireless transmission medium during propagation. A node has the capability of detecting a corrupted received packet and can discard it.

iii. All mobile nodes are homogeneous. The wireless transmission range and the interface card are the same. Likewise the wireless channel is shared by all nodes and can be accessed by any node at random time. Therefore, collision is a possible phenomenon with the channel.

\subsection{Simulation Setup}

We use ns-2 packet level simulator (v.2.29) [10] to simulate a square $600 \mathrm{~m}$ by $600 \mathrm{~m}$ area populated with $25,50,75, \ldots, 150$ mobile nodes that are uniformly distributed in the region, each with a circular radio transmission range of radius $100 \mathrm{~m}$. This corresponds to networks consisting of multi-hops radio across while the selected mobile nodes represent the various network densities ranging from sparse to high density network. The radio propagation model used in this study is the ns-2 default, which uses characteristic similar to a commercial radio interface, Lucent's WaveLAN card with a $2 \mathrm{Mbps}$ bit rate [13]. The distributed coordination function (DCF) of the IEEE 802.11 protocol [14] is utilized as MAC layer protocol while random waypoint model [15] is used as the mobility model. Because it takes time for the random way point model to reach a stable distribution of mobile nodes [16], the modified random waypoint mobility model [15] used take care of this node distribution problem. The simulation is allowed to run for 900 seconds for each simulation scenario. Other simulation parameters that have been used in our experiment are shown in Table 1.

Table 1. Simulation Parameters

\begin{tabular}{|l|l|}
\hline Simulation Parameter & Value \\
\hline Simulator & NS-2 $(\mathrm{v} .2 .29)$ \\
Transmission range & 100 meters \\
Bandwidth & 2 Mbps \\
Interface queue length & 50 \\
Packet size & 512 byte \\
Traffic type & CBR \\
Packet rate & 10 packets $/ \mathrm{sec}$ \\
Topology size & $600 \times 600 \mathrm{~m}^{2}$ \\
Number of nodes & $25,50, \ldots, 150$ \\
Number of trials & 30 \\
Simulation time & $900 \mathrm{sec}$ \\
Maximum speed & $20 \mathrm{~m} / \mathrm{s}$ \\
Counter threshold $(\mathrm{C})$ & 4 \\
RAD Tmax & $0.01 \mathrm{~seconds}$ \\
\hline
\end{tabular}

Each data point represents an average of 30 different randomly generated mobility models with $95 \%$ confidence interval. Likewise, the maximum speed used is the ns-2 default which characterise a high mobility network. 


\subsection{Simulation Results}

In this section, we present the performance results of ECS (efficient counter-based broadcast scheme) side by side with counter-based, fixed probability and flooding. The simulation output is collected using replication mean method where each data point represents an average of 30 different randomly generated mobility models with $95 \%$ confidence intervals. Our main focus is to mitigate the broadcast storm problem therefore reducing the contention in the network and decreasing the probability of packet collisions. As a result, end-to-end delay can be reduced, and the percentage of saved rebroadcast can be improved.

\subsubsection{Saved Rebroadcast (SRB)}

Figure 2 shows the performance comparisons of fixed probability, counter-based, flooding and ECS in terms of SRB with varying network density. The four schemes achieve different SRB percentages with increasing network density. The figure demonstrates that ECS can significantly mitigate the contentions and collisions incur during broadcasting especially in dense networks with node moving at $20 \mathrm{~m} / \mathrm{s}$. In sparse networks, ECS has superior SRB of $46 \%$ and about 56\% in medium and high dense networks. Under the same network conditions, the SRB achieved by the other algorithms are as follows: fixed probability has $39 \%$ and $35 \%$; counter-based has $22 \%$ and $32 \%$; and flooding has $4 \%$ and $1 \%$ for sparse and medium - high dense network respectively. Thus, ECS has superior SRB performance in various network densities. As shown in Figure 2, ECS can substantially reduce the number of rebroadcast because nodes rebroadcast a packet with a certain probability value $(0.65)$ rather than automatically rebroadcasting every received packet. However, sending too few rebroadcast can result in broadcast packet not reaching all the nodes in the network.

\subsubsection{Reachability}

Figure 3 shows that reachability increases when network density increases regardless of which scheme is used. Flooding has best performance in terms of reachability, reaching about $100 \%$ of the nodes. The performance of ECS scheme shows that the reachability is about $95 \%$ in sparse networks and above $98 \%$ in medium and high density network. In high density networks, very similar and comparable results are obtained for all the four schemes. However, in the case of low density networks (specifically 25 nodes), flooding and counter-based schemes achieved better reachability performance than ECS. As redundant rebroadcasts also contribute to chances of packet collisions which may eventually cause packet drops, thus negatively affecting the reachability. Depending on the value of the probability, ECS may have lower reachability compared to flooding and counter-based schemes. However, by choosing appropriate probability value, we can achieve acceptable reachability. ECS 's inferior reachability performance in sparse network is due to fact that the network might be partition and thus increasing the likelihood of more broadcast packets not reaching all the nodes in the network.

\subsubsection{Latency}

In this section we measure the end-to-end delay of the broadcast packet that has been received by all nodes in the network. The results in figure 4 show the effects of 
network density on the latency of broadcast packets. When node density increases, more broadcast packets fail to reach all the nodes due to high probability of packet collision and channel contention caused by excessive redundant retransmission of broadcast packets. Therefore the waiting time of packets in the interface queues increases. As shown in figure 4, ECS exhibits lower latency than counter-based, fixed probability and flooding. Since rebroadcast packets collide and content for channel with each other, and the ECS incurs the lowest number of rebroadcasts (highest saved-rebroadcast), it should have the lowest latency.

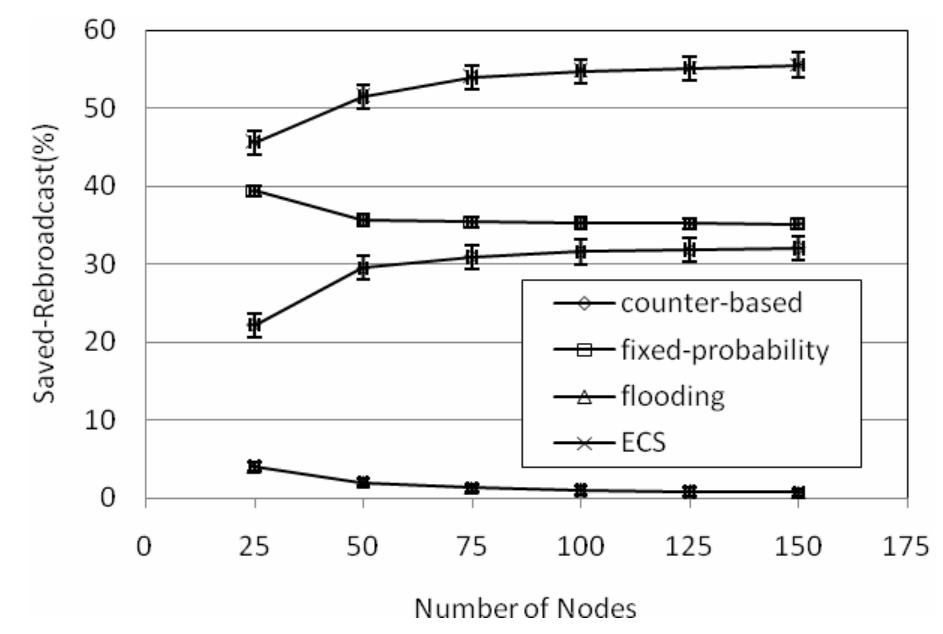

Fig. 2. Saved-Rebroadcast of the four schemes against network density

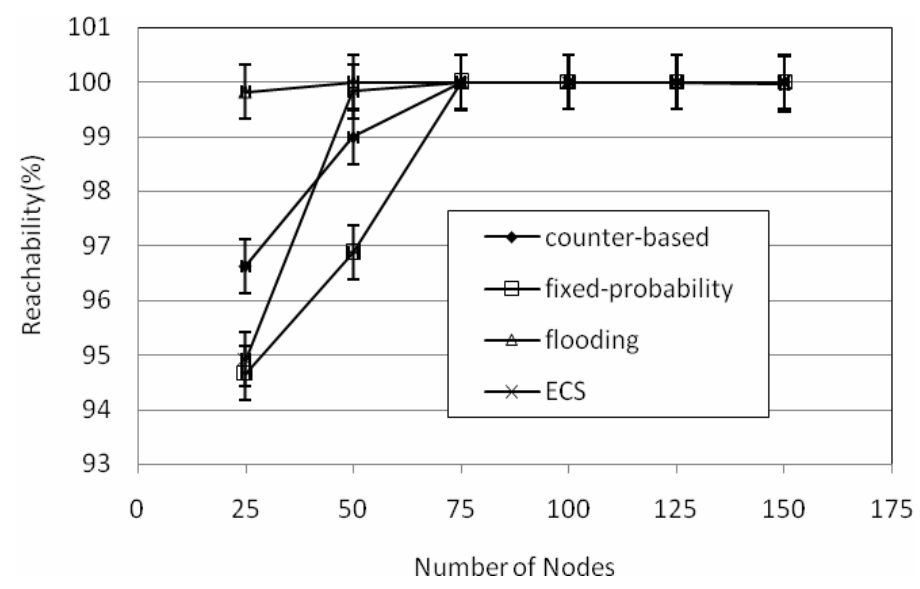

Fig. 3. Reachability of the four schemes against network density 


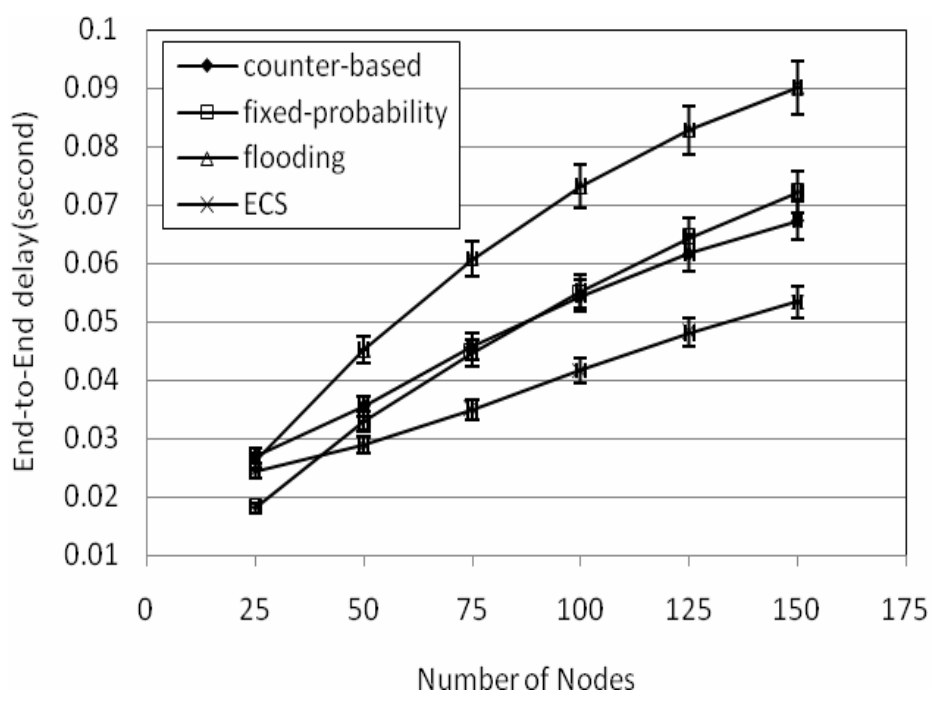

Fig. 4. Latency of the four schemes against network density

\section{Conclusion}

This paper has proposed an efficient counter-based broadcast scheme for MANETs that mitigate the broadcast storm problem associated with flooding. The scheme uses two different probability values to distinguish between rebroadcast probability for nodes in sparse network and that of a dense network. In order to reduce the broadcast overhead and without sacrificing the network connectivity in dense networks, the rebroadcast probability of nodes located in sparse areas is set high and that of nodes located in dense areas is set low. Compared to flooding, fixed probability and counterbased schemes, our simulation results have revealed that the adjusted counter-based scheme can achieve up to $56 \%$ saved rebroadcast without sacrificing reachability in both medium to high density networks. Likewise the scheme has better latency.

As a continuation of this research in the future, we plan to investigate the performance of our scheme under a more realistic scenario (non uniform node distribution) and that achieved by a routing protocol when they employ ECS broadcast schemes. Furthermore, we intend to build an analytical model for our efficient counter-based scheme in order to facilitate its validation strategy.

\section{References}

1. Ni, S., Tseng, Y., Chen, Y., Sheu, J.: The Broadcast Storm Problem in a Mobile Ad Hoc Networks. In: The Broadcast Storm Problem in a Mobile Ad Hoc Networks, pp. 151-162. IEEE Computer Society Press, Los Alamitos (1999)

2. Tseng, Y.-C., Ni, S.-Y., Chen, Y.-S., Sheu, J.-P.: The Broadcast Storm Problem in a Mobile Ad Hoc Network. Wireless Networks. 8, 153-167 (2002) 
3. Tseng, Y.-C., Ni, S.-Y., Shih, E.-Y.: Adaptive Approaches to Relieving Broadcast Storms in a Wireless Multihop Ad Hoc Networks. IEEE Transactions on Computers. 52, 545-557 (2003)

4. Williams, B., Camp, T.: Comparison of Broadcasting Techniques for Mobile Ad Hoc Networks. In: Williams, B., Camp, T. (eds.) Proceeding MOBIHOC., pp. 194-205. Lausanne, Switzerland (2002)

5. Lou, W., Wu, J.: Localized Broadcasting in Mobile Ad Hoc Networks Using Neighbour Designation. CRC Press, Boca Raton, USA (2003)

6. Alireza, K-H., Vinay, R., Rudolf, R.: Color-Based Broadcasting for Ad Hoc Networks. 4th International Symposium on Modeling and Optimization in Mobile, Ad Hoc, and Wireless Networks, pp. 1-10 (2006)

7. Haas, Z.J., Halpern, J.Y., Li, L.: Gossip-based ad hoc routing. In: Proceeding of IEEE INFOCOM, IEEE Computer Society Press, Los Alamitos (2002)

8. Cartigny, J., Simplot, D.: Border node retransmission based probabilistic broadcast protocols in ad hoc networks. Telecommunication Systems. 22, 189-204 (2003)

9. Zhang, Q., Agrawal, D.P.: Dynamic Probabilistic Broadcasting in MANETs. Parallel and Distributed Computing. 65, 220-233 (2005)

10. The Network Simulator ns-2, http://www.isi.edu/nsnam/ns/

11. Perkins, C.E., Moyer, E.M.: Ad-hoc on-demand distance vector routing. In: Proceedings of 2nd IEEE Workshop on Mobile Computing Systems and Applications, pp. 90-100. IEEE Computer Society Press, Los Alamitos (1999)

12. Johnson, D.B., Maltz, D.A.: Dynamic source routing in ad hoc wireless networks. Mobile Computing, pp. 153-181. Dordrecht Academic Publishers, The Netherlands (1996)

13. IEEE802.11 WaveLAN PC Card - User's Guide, A-1

14. Internet Standard Comm: Wireless LAN medium access control (MAC) and physical layer (PHY) specifications. IEEE standard 802.11-1997. IEEE, New York (1997)

15. Navidi, W., Camp, T., Bauer, N.: Improving the accuracy of random waypoint simulation through steady-state initialization. In: Proceedings of the 15th International Conference on Modeling and Simulation (MS'04), Marina Del Rey, Califonia, USA (2004)

16. Camp, T., Boleng, J., Davies, V.: A survey of mobility models for ad hoc network research. Wireless Communication and Mobile Computing (WCMC), vol. 2 (2002)

17. Colagrosso, M.D.: Intelligent broadcasting in mobile ad hoc networks: Three classes of adaptive protocols. EURASIP Journal on Wireless Communication and Networking. 2007, p. 16 (2007) 